\title{
Les relations diplomatiques France-Canada-Québec 50 ans après « Vive le Québec Libre !»
}

Jérémie Cornut

\section{CpenEdition}

\section{Journals}

Édition électronique

URL : https://journals.openedition.org/ideas/1917

DOI : $10.4000 /$ ideas. 1917

ISSN : 1950-5701

Éditeur

Institut des Amériques

Référence électronique

Jérémie Cornut, «Les relations diplomatiques France-Canada-Québec 50 ans après « Vive le Québec Libre ! » », IdeAs [En ligne], 9 | 2017, mis en ligne le 12 juillet 2017, consulté le 18 octobre 2022. URL: http://journals.openedition.org/ideas/1917 ; DOI : https://doi.org/10.4000/ideas.1917

Ce document a été généré automatiquement le 18 octobre 2022.

\section{(c) (i) $\odot$}

Creative Commons - Attribution - Pas d'Utilisation Commerciale - Pas de Modification 4.0 International - CC BY-NC-ND 4.0

https://creativecommons.org/licenses/by-nc-nd/4.0/ 


\title{
Les relations diplomatiques France- Canada-Québec 50 ans après « Vive le Québec Libre!»
}

\author{
Jérémie Cornut
}

1 Le discours de De Gaulle à Montréal en 1967 marque un tournant dans la relation entre le Canada et la France : en prenant une position claire en faveur de l'indépendance du Québec, la France empoisonne ses relations avec Ottawa. Ce «Vive le Québec libre! » a profondément marqué la conscience publique et les gestes symboliques exécutés tous les dix ans pour le commémorer - ce que Frédéric Bastien a qualifié de «culte du souvenir» (Bastien F., 1999 : 335-352) - font en sorte que le slogan est encore frais dans la mémoire collective, en particulier au Canada et au Québec. L'impression qui reste est souvent que la France voit d'un bon œil le mouvement souverainiste, quitte à nuire à ses relations avec Ottawa (voir par exemple Bosher J., 1999).

2 Pourtant, la « relation spéciale » entre la France et le Québec s'est transformée au cours des trois dernières décennies du XX ${ }^{\text {ème }}$ siècle. À partir de 1977, la France a opté pour la politique du «ni-ni », «ni ingérence ni indifférence », inventée par Alain Peyrefitte, conseiller de De Gaulle et ministre de la Justice sous la présidence de Valery Giscard d'Estaing. Cette formule, plus destinée à éviter d'offenser autant Ottawa que Québec qu'à énoncer une politique précise, exprime l'extrême prudence de la France sur la question de la souveraineté québécoise. Si plusieurs facteurs expliquent ce changement, le premier d'entre eux est que Québec n'est pas favorable à l'ingérence française. Cette politique a été formulée à la demande du Parti québécois, arrivé au pouvoir à Québec en 1976 et qui avait des réticences à voir des acteurs extérieurs s'impliquer dans un débat qui ne concerne que les Québécois. En signalant que la France n'interviendrait plus dans le débat fédéral-provincial canadien, cette nouvelle politique est en fait le contraire de la position gaulliste - même si cela n'est pas toujours perçu clairement par les observateurs et les acteurs politiques.

3 Après la démission de De Gaulle, la France n'encourage plus vraiment l'indépendance du Québec. Le Québec, quant à lui, multiplie et diversifie ses relations extérieures, se 
rapprochant d'autres États et entités sous-étatiques tout en gardant la France comme partenaire le plus important. Pour présenter cette évolution, cet article commence par s'intéresser aux relations France-Canada-Québec dans les décennies qui suivent la déclaration de De Gaulle, puis analyse les logiques qui président à ces relations aujourd'hui.

\section{La France « accompagne » le Québec}

Bien que cela soit plus rare aujourd'hui, il était courant au cours des dernières décennies du $\mathrm{XX}^{\text {ème }}$ siècle de voir certains dignitaires français appuyer publiquement la souveraineté québécoise. D'anciens dirigeants français, dont l'ancien Premier ministre Michel Rocard, l'ancien président de l'Assemblée nationale Philippe Séguin et PierreAndré Wiltzer, ancien ministre et ancien président de l'Association France-Québec, prenaient régulièrement position en faveur de la souveraineté québécoise. Immédiatement avant le référendum sur la souveraineté de 1995, un groupe de trente intellectuels français a signé un manifeste favorable à l'indépendance du Québec. Fidèles à l'héritage gaulliste, ces dignitaires français faisaient partie de ce que certains appelaient le « lobby du Québec à Paris ».

Les actions officielles de la France allaient parfois dans le même sens. Elles conféraient certains attributs de la souveraineté au Québec, comme lorsque le président français recevait le premier ministre québécois. Ces actes nuisaient aux relations de la France avec le gouvernement fédéral, qui les percevait comme une menace pour l'unité nationale canadienne. L'invitation faite par la France au Québec à participer à un forum international sur la culture qui s'est tenu à Paris en mars 1999 a par exemple été critiquée par Ottawa. De même, la déclaration de Raymond Barre de 1980 selon laquelle le retrait de la France de la Conférence des représentants francophones de Dakar était justifié au motif que la France ne voulait pas exposer le Québec au "poison de l'indifférence » montre que la France n'avait pas complètement abandonné la politique gaulliste (Poulin P., 2002 : 147).

6 Ceci étant dit, tout au long des dernières décennies du XX ${ }^{\text {ème }}$ siècle, la France a, la plupart du temps, refusé de s'ingérer dans les affaires intérieures canadiennes. Au lieu de favoriser le mouvement souverainiste comme de Gaulle l'avait fait, elle a laissé Ottawa et Québec régler seuls leurs différends. Malgré des déclarations soigneusement étudiées pour n'offenser aucune des parties, son approche était claire - à l'exception des quelques exemples mentionnés ci-dessus. Par exemple, le $450^{\text {ème }}$ anniversaire du premier voyage de Jacques Cartier en Amérique du Nord en 1534 a donné lieu à deux cérémonies différentes à Saint-Malo. Le président français François Mitterrand était très tiède sur la question de la souveraineté québécoise, dans laquelle il voyait une " lubie » de De Gaulle, qu'il avait d'ailleurs publiquement critiqué en 1967 (Bastien 1999 : 181). Autre exemple, en 1997, Paris a accédé à la demande d'Ottawa de ne pas émettre de timbre commémorant le $30^{\text {ème }}$ anniversaire du discours de De Gaulle à Montréal, mettant fin à une saga de plusieurs semaines surnommée par la presse la «guerre du timbre ».

7 En effet, aucun des dignitaires qui forment le «lobby du Québec à Paris » n'était membre du corps diplomatique français au moment d'apporter son soutien à un Québec indépendant. La politique étrangère de la France est traditionnellement le « domaine réservé » du président, qui, en collaboration avec le ministre des Affaires étrangères, 
s'y intéresse de très près. Seules ces voix déterminent la politique officielle de la France, et les opinions exprimées par d'autres n'expriment que des points de vue personnels. Il n'était ainsi pas rare de voir les dignitaires français prendre une position moins favorable à l'indépendance du Québec une fois qu'ils étaient arrivés aux commandes de la politique étrangère de la France.

8 Cela n'empêchait pas la France de maintenir les apparences d'un appui spécial au Québec. Par exemple, Jacques Parizeau a effectué une visite officielle en France début 1995 afin de relancer la coopération franco-québécoise et de déterminer quelle serait la position de la France face à un Québec souverain. Selon sa stratégie - surnommée le "grand jeu "-, une reconnaissance rapide par la France aurait un effet domino qui permettrait une reconnaissance de la part des pays de la Francophonie et des ÉtatsUnis. La France était elle-même au milieu d'une campagne électorale qui voyait les deux rivaux, Jacques Chirac et Édouard Balladur (alors Premier ministre) engagés dans une bataille féroce pour endosser l'héritage gaulliste. Dans ce contexte, les deux hommes ont jugé stratégique de se prononcer en faveur d'un Québec souverain. Balladur a déclaré qu'il était prêt à suivre les recommandations de Parizeau, alors que Chirac a estimé qu'en cas de vote "oui », la France reconnaîtrait le Québec, ce qui aboutirait à la reconnaissance des autres membres de la Francophonie. Comme le souligne Bastien, la question de la souveraineté québécoise était exploitée par les factions politiques françaises dans une tentative de marquer des points politiques sur le front intérieur (Bastien F., 1999 : 303-314). Valéry Giscard d'Estaing et Jacques Chirac avaient également utilisé la question québécoise comme un terrain d'affrontement à la fin des années 1970.

Lors de la campagne référendaire de 1995 elle-même, les dirigeants français ont déclaré que « quelle que soit la route choisie par le Québec, la France l'accompagnerait». Lors d'une entrevue sur $\mathrm{CNN}$ peu avant le référendum, Chirac élu président considérait ainsi que «si le oui gagne, la France reconnaîtra ce fait». En réalité, ces déclarations ne faisaient que reformuler la politique de Giscard d'Estaing de 1977. Pour clarifier la politique du «ni-ni », que René Lévesque considérait trop vague, Giscard d'Estaing a expliqué qu'il voulait accompagner le Québec: «La France devrait sans doute figurer parmi les premiers à dire au Québec que nous marcherons à ses côtés [...]. Les États francophones, et en particulier la France, devraient être immédiatement du côté des Québécois et reconnaître la nouvelle situation » (Beaudoin L., 2006). Comme le souligne Louise Beaudoin, bien que différentes variantes de cette métaphore de l'accompagnement aient été formulées depuis 1977 selon la personnalité et la tendance politique de l'énonciateur, la substance est restée la même.

La politique québécoise de la France lors du référendum de 1995 est donc essentiellement inchangée. En «accompagnant » le Québec, la France n'interfère pas dans les affaires intérieures du Canada. Au lieu d'essayer d'accélérer la "libération » québécoise comme elle l'avait fait en 1967, elle attend la décision du Québec sur une question qui ne peut être tranchée que par les Québécois. Les déclarations françaises annonçant la reconnaissance des résultats du référendum permettent dans une certaine mesure aux souverainistes d'apaiser les inquiétudes du public quant à l'isolement possible d'un Québec souverain. Mais dans l'ensemble, la position de la France n'était pas particulièrement audacieuse, ni différente de celle des autres pays. Cette politique paraît d'autant moins risquée que depuis l'échec du premier référendum de 1980, les diplomates français prédisent - comme le fait Chirac sur CNN 
en 1995 - que les Québécois allaient voter "non» à la souveraineté, ce que confirmaient les sondages.

\section{Le triangle Paris-Ottawa-Québec aujourd'hui}

11 La France a ainsi progressivement abandonné la politique gaulliste au cours des dernières décennies du XX ${ }^{\text {ème }}$ siècle et trois évolutions structurent les relations FranceCanada-Québec aujourd'hui. Premièrement, le gouvernement fédéral ne perçoit plus les liens du Québec avec la France comme une menace à l'unité nationale du Canada. Ottawa promeut même ces liens avec la France et d'autres États et entités sousétatiques. Ainsi, l'assouplissement des relations fédérales-provinciales a permis le développement de la Francophonie dans les années 1980. La France, pour sa part, ne craint plus que de bonnes relations avec le Québec nuisent à ses relations avec le Canada au sein du G7, à l'OTAN ou à l'ONU. Les nombreux « accords » que la France conclut avec le Québec relèvent généralement d'un accord "parapluie » signé avec le Canada. La France « soutient la Francophonie en Amérique du Nord sans se mêler à un conflit interne à la fédération canadienne » (Tenzer N.et Rouah E., 1987 : 72).

Deuxièmement, les relations entre la France et le Québec ne dépendent plus de la ligne politique du parti au pouvoir au Québec. Bien sûr, l'arrivée d'un gouvernement souverainiste peut avoir un effet revigorant sur ces relations. Par exemple, alors que les réunions alternées des premiers ministres ont rarement eu lieu de 1985 à 1994 lorsque le gouvernement libéral de Robert Bourassa était au pouvoir, elles ont été réintroduites au moment où Parizeau est entré en fonction en 1994. Il faut toutefois souligner que Jean Charest, à la tête d'un gouvernement non souverainiste de 2003 à 2012, a activement cultivé la relation France-Québec, notamment en favorisant les échanges économiques. La participation du Premier ministre français Jean-Pierre Raffarin et du Premier ministre Charest à la mission commerciale conjointe France-Québec au

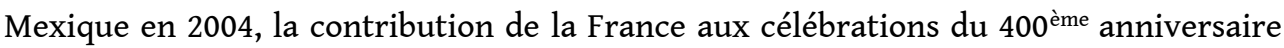
de la fondation de Québec, la signature en 2008 d'un accord sur la mobilité de la maind'œuvre entre la France et le Québec et les négociations d'un accord de libre-échange entre le Canada et l'UE montrent que les souverainistes n'ont pas le monopole des relations étroites avec la France.

13 Troisièmement, le fait que le Québec ne dépend pas de la diplomatie française lui permet d'établir des liens solides avec les États et les entités sous-étatiques en dehors de la sphère d'influence française et de poursuivre ses intérêts sans crainte de l'opposition française. L'autonomie à l'égard de la France était indispensable à l'expansion d'une diplomatie québécoise respectée sur la scène internationale. Ainsi, au cours des années 1980, le Québec a appuyé l'accord de libre-échange entre le Canada et les États-Unis, malgré les réserves de la France. En outre, comme le montre le différend sur le doublage des films en français, les deux États sont en compétition dans le domaine des produits culturels pour le marché francophone. De même, Québec et Ottawa ont dénoncé l'interdiction de l'amiante décidée par la France en 1997, une question économique importante pour le Québec. Cette critique n'a toutefois pas empêché la France d'essayer avec succès de convaincre d'autres pays européens de suivre son exemple en interdisant le minerai.

14 C'est ainsi que le triangle France-Canada-Québec s'est normalisé au cours des dernières décennies $d u X X^{\text {ème }}$ siècle. Cela invite à relativiser la rupture entraînée par la 
présidence de Nicolas Sarkozy. Il a beaucoup été dit que le successeur de Jacques Chirac avait rompu avec la politique traditionnelle de la France vis-à-vis du Québec, en adoptant une position résolument fédéraliste. En réalité, l'approche de Sarkozy n'était pas en totale contradiction avec l'attentisme de ses prédécesseurs depuis plus de trente ans. C'est en fait la formulation de la politique du « ni-ni » à la fin des années 1970 qui a constitué le véritable changement par rapport à l'approche de De Gaulle. Bien que le ton des déclarations de Sarkozy, en particulier ses éloges de l'unité nationale du Canada, contraste avec celui de ses prédécesseurs, depuis le début des années 1980, la non-ingérence - donc le soutien à la structure fédérale existante - est de fait ce qui caractérise la position de la France vis-à-vis du Québec.

Le président François Hollande s'inscrit dans la même lignée. Ses déclarations sont restées très mesurées et il n'utilise pas lui-même directement la formule «ni ingérence, ni indifférence ». Cela pourrait être anecdotique, si son ministre des Affaires étrangères Laurent Fabius n'avait pas lui aussi évité d'utiliser cette formule lors de sa rencontre avec John Baird, son homologue canadien, en octobre 2012. Ce sont là des signes que la « non-ingérence » a définitivement pris le dessus sur la « non-indifférence ».

\section{BIBLIOGRAPHIE}

Bastien, Frédéric, Relations particulières: la France face au Québec après de Gaulle, Montréal, Éditions du Boréal, 1999.

Beaudoin, Louise, « Un appui renouvelé au Québec », La Presse, 22 mars 2006.

Bosher, John, The Gaullist attack on Canada, 1967-1997, Montréal, McGill-Queen's University Press, 1999.

Poulin, Philippe, « France-Québec: quarante ans de relations 'directes et privilégiées' », Bulletin d'histoire politique, vol. 10, $\mathrm{n}^{\circ}$ 2, 2002, p. 144-149.

Tenzer, Nicolas et Rouah, Éric, « Entre le Canada et la France, de nouveaux liens privilégiés », Défense Nationale, vol. 43, 1987, p. 69-82.

\section{AUTEUR}

\section{JÉRÉMIE CORNUT}

Jérémie Cornut est professeur au département de science politique de l'Université Simon Fraser à Vancouver au Canada. Ses enseignements et sa recherche portent sur la diplomatie, la politique étrangère canadienne et la théorie des relations internationales. Avant d'être à l'Université Simon Fraser, il a occupé des postes de recherche à l'université McGill, la Balsillie School of International Affairs, l'University of Southern California et l'Université du Québec à Montréal. jcornut@sfu.ca 